\title{
Robust Multiple Constrained State Feedback Controller Design with Minimum Auxiliary Entropy for Linear Discrete Systems
}

Wen-Jer Chang

Associate Professor Department of Marine Engineering and Technology, National Taiwan Ocean Universiry. Keelung 20224. Taiwan.R.O.C., wjchang@ntou66.ntou.edu.tw

Follow this and additional works at: https://jmstt.ntou.edu.tw/journal

Part of the Engineering Commons

\section{Recommended Citation}

Chang, Wen-Jer (1998) "Robust Multiple Constrained State Feedback Controller Design with Minimum Auxiliary Entropy for Linear Discrete Systems," Journal of Marine Science and Technology. Vol. 6: Iss. 1, Article 10. DOI: $10.51400 / 2709-6998.2523$

Available at: https://jmstt.ntou.edu.tw/journal/vol6/iss1/10

This Research Article is brought to you for free and open access by Journal of Marine Science and Technology. It has been accepted for inclusion in Journal of Marine Science and Technology by an authorized editor of Journal of Marine Science and Technology. 


\section{Robust Multiple Constrained State Feedback Controller Design with Minimum Auxiliary Entropy for Linear Discrete Systems}

\section{Acknowledgements}

The author would like to express his sincere gratitude to two anonymous reviewers who gave me some constructive comments, cirticisms and suggestions. This work was supported by the National Science Council of the Republic of China, under contract NSC87-2213-E-019-012. 


\title{
ROBUST MULTIPLE CONSTRAINED STATE FEEDBACK CONTROLLER DESIGN WITH MINIMUM AUXILIARY ENTROPY FOR LINEAR DISCRETE SYSTEMS
}

\author{
Wen-Jer Chang*
}

Keywords: State feedback control, Auxiliary entropy, $\mathbf{H}_{\infty}$ norm, State variances, Regional pole assignment.

\section{ABSTRACT}

Existence of a state feedback controller with minimized auxiliary entropy design problem will be explained in this paper. This state feedback controller will achieve multiple performance constraints for linear discrete systems. These constraints include individual state variance constraints, $\mathbf{H}_{\infty}$ norm constraints and regional pole assignment constraints. By achieving the previous three requirements, the state feedback controller can then be solved through use of the singular value decomposition (SVD) techniques and the theory of generalized inverses. An example will be provided to demonstrate the effectiveness of the present methodology.

\section{INTRODUCTION}

The controller design approach, which minimizes an entropy integral, is outlined in monographs [1-4]. As shown in [1], these controllers lie between $\mathbf{H}_{\infty}$ optimal controllers and LQG optimal controllers. Controllers which minimize entropy are considered optimal because of the risk-sensitive control problem of the stochastic control theory [5]. Many direct extensions [1-4, 6-9] result from similarities between the control and the classical LQG control. Under a minimal set of assumptions, the combined $\mathbf{H}_{2} / \mathbf{H}_{\infty}$ approach [1-4, 6-8] gives formulas for solving the problem of finding a controller such that an upper bound for the generalized $\mathbf{H}_{2}$ norm of a closed-loop transfer matrix and the $\mathbf{H}_{\infty}$ norm of some other closedloop transfer matrix are jointly below specified

Paper Received March, 1998. Revised April, 1998. Accepted May, 1998. Author for Correspondence: Wen-Jer Chang.

*Associate Professor Department of Marine Engineering and Technology, National Taiwan Ocean University, Keelung 20224, Taiwan. R.O.C.E-mail: wjchang@ntou66.ntou.edu.tw levels. In [9], it is shown that the Linear Matrix Inequality (LMI) approach is a convenient tool for expressing and combining a variety of design specifications, including performance tracking, disturbance rejection, LQG performance, robustness to model uncertainty, and pole placement objectives.

It is well known that pole location is directly associated with performance specifications such as the setting time and rise time of a control system. In practice, the exact pole location is not required and it is often enough to simply locate them in a prescribed region in the left half-complex plane for continuous time systems [10-12], or in the unit disk for discrete time systems [11-14]. A well known desired region for linear discrete systems is a disk $D$ centered $(\mu, \mathbf{0})$ with radius $\rho$, where $\rho<1$ and $|\mu|+\rho \leq 1$. The problem of locating all the closed-loop poles of controlled linear discrete systems inside this specified disk $D(\mu, \rho)$ is explained in the literature [11-14].

In $[1-4,9-10,12]$, an optimization procedure is provided to achieve pole placement constraints and $\mathbf{H}_{2} / \mathbf{H}_{\infty}$ norm constraints. As pointed out by Skelton [15], the optimal control technique can only guarantee that the entire control system state vector behaves well. Little can said about the transient behavior of individual state variables. The Riccati-like equation approach applied in [1-13], which minimizes a quadratic cost function, lacks the guaranteed individual variance constraints with respect to system state. To overcome such drawbacks, the authors developed an upper bound covariance control technique [16-19] which achieves individual variance constraints and performs good transient behavior of the individual state variables. In the previous exhibits [16-19], the minimum auxiliary entropy and pole assignment constraints are not considered. Additional numerical steps (e.g., SVD) are required in the controllers of [16-19]. These additional steps prevent the control- 
lers from obtaining a precise in closed form. Hence, the purpose of this paper is to develop a methodology to design state feedback controllers such that closedloop discrete-time systems simultaneously satisfy $\mathbf{H}_{\infty}$ norm constraints, circular pole constraints (i.e., $D(\mu, \rho)$ stability), individual state variance constraints and minimum auxiliary entropy. The first contribution of the present approach is to improve the previous treatments [16-19]. It will characterize a closed form set of state feedback controllers that allow the controller parameterization to be explicit in the parameters of the plant. Next, the present approach based on the upper bound covariance control technique [16-19] will provide good individual state transient behavior rather than the mixed $\mathbf{H}_{2} / \mathbf{H}_{\infty}$ method [1-8] and LMI method [9]. A numerical example will illustrate the theoretical results of this approach.

\section{PERFORMANCE REQUIREMENTS DESCRIPTION}

Consider a linear discrete system described by

$$
\begin{aligned}
& \boldsymbol{x}(\boldsymbol{k}+1)=\mathbf{A} \boldsymbol{x}(\boldsymbol{k})+\mathbf{B} \boldsymbol{u}(\boldsymbol{k})+\mathbf{D} w(k), \\
& \boldsymbol{y}(\boldsymbol{k})=\boldsymbol{x}(\boldsymbol{k}), \\
& u(k)=G y(k),
\end{aligned}
$$

where $\boldsymbol{x}(\boldsymbol{k}) \in \mathbf{R}^{\mathbf{n}_{\mathbf{x}}}, \mathbf{A} \in \mathbf{R}^{\mathbf{n}_{\mathbf{x}} \times \mathbf{n}_{\mathbf{x}}}, \mathbf{B} \in \mathbf{R}^{\mathbf{n}_{\mathbf{x}} \times \mathbf{n}_{\mathbf{u}}}, \mathbf{D} \in \mathbf{R}^{\mathbf{n}_{\mathbf{x}}}$ $\times \mathbf{n}_{\mathbf{w}}, \mathbf{G} \in \mathbf{R}^{\mathbf{n}_{\mathbf{u}} \times \mathrm{n}_{\mathbf{u}}}$ and $\boldsymbol{w}(\boldsymbol{k})$ is a zero mean Gaussian white noise process with covariance I. Furthermore, $\boldsymbol{w}(\boldsymbol{k})$ and $\boldsymbol{x}(\boldsymbol{0})$ are independent of each other. It is easy to find that the closed-loop system has the following form

$$
\begin{aligned}
& x(k+1)=\hat{\mathbf{A}} x(k)+\mathbf{D} w(k), \\
& y(k)=x(k),
\end{aligned}
$$

where $\hat{\mathbf{A}} \equiv \mathbf{A}+\mathbf{B G}$. For the closed-loop system (2), the closed-loop transfer function $\mathbf{H}(\mathbf{z})$ from noise input $\boldsymbol{w}(\boldsymbol{k})$ to output $\boldsymbol{y}(\boldsymbol{k})$ may be written as

$$
\mathbf{H}(\mathbf{z})=(\mathbf{z I}-\hat{\mathbf{A}})^{-1} \mathbf{D} \text {. }
$$

\section{Definition 1}

For a given positive scalar $\gamma$, let the closed-loop transfer function $\mathbf{H}(\mathbf{z})$ satisfy $\|\mathbf{H}(\mathbf{z})\|_{\infty} \leq \gamma$, where $\|\mathbf{H}(\mathbf{z})\|_{\infty} \equiv \sup _{\theta \in[0,2 \pi]} \sigma_{\max }\left[\boldsymbol{H}\left(\boldsymbol{e}^{j \theta}\right)\right]$ and $\sigma_{\max }(\bullet)$ denotes the maximum singular value of $(\bullet)$. Then the entropy of $\mathbf{H}(\mathbf{z})$ can be defined as follows [4].

$$
\begin{aligned}
\boldsymbol{I}(\boldsymbol{H}, \gamma) & \equiv-\frac{\gamma^{2}}{2 \pi} \int_{-\pi}^{\pi} \ln \left|\operatorname{det}\left(\boldsymbol{I}-\gamma^{-2} \boldsymbol{H}^{T}\left(e^{-j \theta}\right) \boldsymbol{H}\left(\boldsymbol{e}^{j \theta}\right)\right)\right| d \theta \\
& =-\gamma^{2} \ln \operatorname{det}\left(\boldsymbol{I}-\gamma^{-2} \hat{\boldsymbol{X}}\right),
\end{aligned}
$$

where $\hat{\boldsymbol{X}} \geq 0$ is the solution of the following Riccatilike equation;

$$
\hat{X}=\hat{A} \hat{X} \hat{A}^{T}+\hat{A} \hat{X}\left(\gamma^{2} I-\hat{X}\right)^{-1} \hat{X} \hat{A}^{T}+D D^{T} .
$$

\section{Proposition 1}

If there exists a positive semi-definite matrix $\overline{\boldsymbol{X}}$ satisfying $\overline{\boldsymbol{X}} \geq \widehat{\boldsymbol{X}}$ and $\boldsymbol{I}-\gamma^{-2} \overline{\boldsymbol{X}}>0$, then we can define the upper bound entropy of $\boldsymbol{I}(\boldsymbol{H}, \gamma)$ as

$$
\boldsymbol{I}_{\boldsymbol{u}}(\boldsymbol{H}, \boldsymbol{\gamma}) \equiv-\gamma^{2} \ln \operatorname{det}\left(\boldsymbol{I}-\gamma^{-2} \overline{\boldsymbol{X}}\right) .
$$

That is, $\boldsymbol{I}_{u}(\boldsymbol{H}, \gamma) \equiv-\gamma^{2} \ln \operatorname{det}\left(\boldsymbol{I}-\gamma^{-2} \overline{\boldsymbol{X}}\right) \geq-\gamma^{2} \ln \operatorname{det}(\boldsymbol{I}$ $\left.-\gamma^{-2} \widehat{X}\right)=\boldsymbol{I}(\boldsymbol{H}, \gamma)$. \#

This proposition gives auxiliary entropy of $\boldsymbol{I}(\boldsymbol{H}$, $\gamma)$ for the closed-loop system (2). The proof of this proposition may be found in the Appendix 2 of [4].

Now, we define $\mathbf{X}$ as the steady state covariance matrix of the state vector of the closed-loop system (2), i.e.,

$$
\mathbf{X} \equiv \lim _{k \rightarrow \infty} \mathbf{E}\left[x(k) x(k)^{T}\right],
$$

where $\mathbf{E}[\bullet]$ denotes the expectation of $[\bullet]$. It is easy to see that $\mathbf{X}$ satisfies the following Lyapunov equation

$$
\mathbf{X}=\hat{\mathbf{A}} \mathbf{X} \hat{\mathbf{A}}^{\mathbf{T}}+\mathbf{D} \mathbf{D}^{\mathbf{T}}
$$

\section{Definition 2}

Consider closed-loop linear discrete system (2). It is said to be $D(\mu, \rho)$-stable if all the eigenvalues $\lambda_{i}$ of matrix $\hat{\mathbf{A}}$ satisfy $\left|\lambda_{i}-\mu\right| \leq \rho$. \#

\section{Lemma 1}

Consider the closed-loop system (2). Let $\mathbf{G}$ be given and let $\gamma>0$ be a fixed scalar. If there exists a positive define matrix $\overline{\mathbf{X}}$ satisfying

$$
\begin{gathered}
2 \hat{\mathbf{A}} \overline{\mathbf{X}} \hat{\mathbf{A}}^{\mathbf{T}}-\mu \hat{\mathbf{A}} \overline{\mathbf{X}}-\mu \overline{\mathbf{X}} \hat{\mathbf{A}}^{\mathbf{T}}+\left(\mu^{2}-\rho^{2}\right) \overline{\mathbf{X}} \\
+\hat{\mathbf{A}} \overline{\mathbf{X}} \mathbf{M} \overline{\mathbf{X}} \hat{\mathbf{A}}^{\mathbf{T}}+\mathbf{D} \mathbf{D}^{\mathbf{T}}=0,
\end{gathered}
$$

where $\mathbf{M}=\left(\gamma^{2} \mathbf{I}-\overline{\mathbf{X}}\right)^{-1}$ is a positive definite matrix, then the closed-loop system (2) is $\boldsymbol{D}(\mu, \rho)$-stable, $\|\mathbf{H}(\mathbf{z})\|_{\infty} \leq \gamma$ and the state covariance matrix satisfies 
$\mathbf{X} \leq \overline{\mathbf{X}}$

proof:

It is assumed that $\lambda$ are $v$ eigenvalue and eigenvector of $\hat{\mathbf{A}}^{\mathbf{T}}$, respectively. Let $\lambda=\boldsymbol{x}+\boldsymbol{j} \boldsymbol{y}$ and put it into (9). We now have

$$
\begin{aligned}
& \left(\boldsymbol{x}^{2}+\boldsymbol{y}^{2}-2 \mu \boldsymbol{x}+\mu^{2}-\rho^{2}\right) v^{\mathbf{T}} \overline{\mathbf{X}} v \\
& =-\left(\boldsymbol{x}^{2}+\boldsymbol{y}^{2}\right) v^{\mathbf{T}} \overline{\mathbf{X}} v-\left(\boldsymbol{x}^{2}+\boldsymbol{y}^{2}\right) v^{\mathbf{T}} \mathbf{X} \mathbf{M} \overline{\mathbf{X}} v-v^{\mathbf{T}} \mathbf{D} D^{\mathbf{T}} v
\end{aligned}
$$

Since the right-hand side of equation (10) is negative definite and we have

$$
(x-\mu)^{2}+y^{2}<\rho^{2},
$$

which means that all eigenvalues of should be located in a specified disk $\boldsymbol{D}(\mu, \rho)$.

Adding to both sides of (9), then equation (9) becomes

$$
\overline{\mathbf{K}}=\overline{\mathbf{X}}-\hat{\mathbf{A}} \overline{\mathbf{X}} \overline{\mathbf{A}}^{\mathbf{T}}-\hat{\mathbf{A}} \overline{\mathbf{X}} \mathbf{M} \overline{\mathbf{X}} \hat{\mathbf{A}}^{\mathbf{T}}-\mathbf{D} \mathbf{D}^{\mathbf{T}},
$$

where $\overline{\mathbf{K}} \equiv(\hat{\mathbf{A}} \overline{\mathbf{Q}}-\mu \overline{\mathbf{Q}})(\hat{\mathbf{A}} \overline{\mathbf{Q}}-\mu \overline{\mathbf{Q}})^{\mathbf{T}}+\left(1-\rho^{2}\right) \overline{\mathbf{X}}$ and $\overline{\mathbf{X}} \equiv \overline{\mathbf{Q}} \overline{\mathbf{Q}}^{\mathbf{T}}$. Note that $\left(1-\rho^{2}\right) \geq 0$ resulting in $\overline{\mathbf{K}} \geq 0$. Let $z=e^{j \theta}$ and $\bar{z}=e^{-j \theta}$, where $\theta \in[\mathbf{0}, 2 \pi]$. Equation (12) is equivalent to

$$
\begin{aligned}
\mathbf{D D}^{\mathbf{T}} & =(z \mathbf{I}-\hat{\mathbf{A}}) \overline{\mathbf{X}}(\bar{z} \mathbf{I}-\hat{\mathbf{A}})^{\mathbf{T}}+\hat{\mathbf{A}} \overline{\mathbf{X}}(\bar{z} \mathbf{I}-\hat{\mathbf{A}})^{\mathbf{T}} \\
& +(z \mathbf{I}-\hat{\mathbf{A}}) \overline{\mathbf{X}} \hat{\mathbf{A}}^{\mathbf{T}}-\hat{\mathbf{A}} \overline{\mathbf{X}} \mathbf{M} \overline{\mathbf{X}} \hat{\mathbf{A}}^{\mathbf{T}}-\overline{\mathbf{K}},
\end{aligned}
$$

or

$$
\begin{aligned}
& (z \mathbf{I}-\hat{\mathbf{A}})^{-1} \mathbf{D D}^{\mathrm{T}}(\bar{z} \mathbf{I}-\hat{\mathbf{A}})^{-\mathbf{T}}=\overline{\mathbf{X}}+(z \mathbf{I}-\hat{\mathbf{A}})^{-1} \hat{\mathbf{A}} \overline{\mathbf{X}} \\
& +\overline{\mathbf{X}} \overline{\mathbf{A}}^{\mathbf{T}}(\bar{z} \mathbf{I}-\hat{\mathbf{A}})^{-\mathbf{T}}-(z \mathbf{I}-\hat{\mathbf{A}})^{-1} \hat{\mathbf{A}} \overline{\mathbf{X}} \mathbf{M} \overline{\mathbf{X}} \overline{\mathbf{A}}^{\mathbf{T}}(\bar{z} \mathbf{I}-\hat{\mathbf{A}})^{-\mathbf{T}} \\
& -(z \mathbf{I}-\hat{\mathbf{A}})^{-1} \overline{\mathbf{K}}(\bar{z} \mathbf{I}-\hat{\mathbf{A}})^{-\mathbf{T}} .
\end{aligned}
$$

Multiply (14) by -1 and add $\gamma^{2} \mathbf{I}$ to both sides of (14). This yields

$$
\begin{aligned}
& \gamma^{2} \mathbf{I}-\mathbf{H}(z) \mathbf{H}^{*}(z)=\left[\mathbf{M}^{-1 / 2}-(z \mathbf{I}-\hat{\mathbf{A}})^{-1} \hat{\mathbf{A}} \overline{\mathbf{X}} \mathbf{M}^{1 / 2}\right] \\
& {\left[\mathbf{M}^{-1 / 2}-(z \mathbf{I}-\hat{\mathbf{A}})^{-1} \hat{\mathbf{A}} \overline{\mathbf{X}} \mathbf{M}^{1 / 2}\right]^{*}} \\
& +(z \mathbf{I}-\hat{\mathbf{A}})^{-1} \overline{\mathbf{K}}(\bar{z} \mathbf{I}-\hat{\mathbf{A}})^{-\mathbf{T}} .
\end{aligned}
$$

It is clear that the right-hand side of equation (15) is positive semi-definite, resulting in $\gamma^{2} \mathbf{I}$ $\mathbf{H}(\mathbf{z}) \mathbf{H}(\mathbf{z})^{*} \geq \mathbf{0}$. This gives $\|\mathbf{H}(\mathbf{z})\|_{\infty} \leq \gamma$.

Subtracting (8) from (12) will give the result of

$$
\begin{aligned}
\overline{\mathbf{X}}-\mathbf{X} & =\hat{\mathbf{A}}(\overline{\mathbf{X}}-\mathbf{X}) \hat{\mathbf{A}}^{\mathbf{T}}+\hat{\mathbf{A}} \overline{\mathbf{X}} \mathbf{M} \overline{\mathbf{X}} \hat{\mathbf{A}}^{\mathbf{T}}+(\hat{\mathbf{A}} \overline{\mathbf{Q}} \\
& -\mu \overline{\mathbf{Q}})(\hat{\mathbf{A}} \overline{\mathbf{Q}}-\mu \overline{\mathbf{Q}})^{\mathbf{T}}+\left(1-\rho^{2}\right) \overline{\mathbf{X}},
\end{aligned}
$$

which is equivalent to

$$
\begin{aligned}
\overline{\mathbf{X}}-\mathbf{X} & =\sum_{\mathbf{i}=0}^{\infty}\left(\hat{\mathbf{A}}^{\mathbf{i}}\right)\left[\hat{\mathbf{A}} \overline{\mathbf{X}} \mathbf{M} \overline{\mathbf{X}} \hat{\mathbf{A}}^{\mathbf{T}}+(\hat{\mathbf{A}} \overline{\mathbf{Q}}-\mu \overline{\mathbf{Q}})(\hat{\mathbf{A}} \overline{\mathbf{Q}}-\mu \overline{\mathbf{Q}})^{\mathbf{T}}\right. \\
& \left.+\left(1-\rho^{2}\right) \overline{\mathbf{X}}\right]\left(\hat{\mathbf{A}}^{\mathbf{i}}\right)^{\mathbf{T}},
\end{aligned}
$$

since $\widehat{\mathbf{A}}$ is stable. Because $\hat{\mathbf{A}} \overline{\mathbf{X}} \mathbf{M} \overline{\mathbf{X}} \hat{\mathbf{A}}^{\mathbf{T}}+(\hat{\mathbf{A}} \overline{\mathbf{Q}}-\mu \overline{\mathbf{Q}})(\hat{\mathbf{A}} \overline{\mathbf{Q}}$ $-\mu \overline{\mathbf{Q}})^{\mathbf{T}}+\left(1-\rho^{2}\right) \geq 0, \overline{\mathbf{X}} \geq \mathbf{X}$ is thus proved.

From Lemma 1, we can conclude that the minimum auxiliary entropy state feedback controller design problem considered in this paper is to determine the state feedback controller $\mathbf{G}$ such that the following performance criteria are satisfied:

$<1>$ Individual variance constraints: $[\mathbf{X}]_{i i} \leq \sigma_{i}^{2}, i=1$,

$2 \ldots, n_{x}$; where $[\bullet]_{i j}$ denote the $i$ th diagonal element of matrix [•] and $\sigma_{i}^{2}$ denotes the Root-Mean-Squared (RMS) constraints for the individual variances of the closed-loop linear discrete system (2).

$<2>\mathbf{H}_{\infty}$ norm constraints: $\|\mathbf{H}(\mathbf{z})\|_{\infty} \leq \gamma$ for some prescribed positive constant $\gamma$.

$<3>D(m, \rho)$-stability constraints: $\lambda(\widehat{\mathbf{A}}) \in D(\mu, \rho)$, where $\lambda(\bullet)$ denotes any eigenvalues of matrix $[\bullet]$. $<4>$ Minimum auxiliary entropy: The auxiliary entropy $\boldsymbol{I}_{u}(\boldsymbol{H}, \gamma)$ defined in (6) is minimized.

\section{STATE FEEDBACK CONTROLLER DESIGN WITH MULTIPLE CONSTRAINTS}

In this section, the minimum auxiliary entropy state feedback controller which satisfies the performance constraints $<1>-<4>$ will be derived for linear discrete systems. First, we will design a state feedback controller to achieve constraints $<1>-<3>$. Theorem 1 will introduce the conditions for the existence of this state feedback controller, which assigns a specified upper bound covariance matrix for equation (9). In Theorem 2, we will find the set of state feedback controller solutions for the specified upper bound covariance matrix as defined in Theorem 1. The necessary conditions for this state feedback controller minimizing auxiliary entropy will be derived in Theorem 3. The Singular Value Decomposition (SVD) theory, theory of generalized inverse, and Lagrange multiplier technique will be used to derive the results of the following theorems.

\section{Definition 3}

Given a desired circular region $D(\mu, \rho)$ and a positive constant $\gamma$, let $\overline{\mathbf{X}}=\overline{\mathbf{X}}^{\mathrm{T}}>0$ be a prespecified positive definite matrix, which meets performance constraint $\langle 1\rangle$. The $\overline{\mathbf{X}}$ is then called a $D-\gamma$-assignable matrix if there exists a set of matrices $\mathbf{G}$ such that equation (9) has the positive definite solution $\overline{\mathbf{X}}$. \#

\section{Theorem 1}


A specified positive definite upper bound covariance matrix $\overline{\mathbf{X}}=\overline{\mathbf{X}}^{\mathbf{T}}>0$ satisfying performance constraint $<1>$ is $D$ - $\gamma$-assignable by some $\mathbf{G}$ if and only if the matrix

$$
\Gamma=\overline{\mathbf{X}}_{\mu \rho}+(\mathbf{A} \overline{\mathbf{R}}-\mu \overline{\mathbf{X}}) \overline{\mathbf{R}}^{-1}\left(\overline{\mathbf{R}} \mathbf{A}^{T}-\mu \overline{\mathbf{X}}\right)
$$

satisfies

(a) $\Gamma \geq 0$, and

(b) $\left(\mathbf{I}-\mathbf{B B}^{+}\right)\left[\Gamma-(\mathbf{A} \overline{\mathbf{R}}-\mu \overline{\mathbf{X}}) \overline{\mathbf{R}}^{-1}\left(\overline{\mathbf{R}} \mathbf{A}^{\mathrm{T}}-\mu \overline{\mathbf{X}}\right)\right]\left(\mathbf{I}-\mathbf{B} \mathbf{B}^{+}\right)$ $=0$,

where $\overline{\mathbf{R}} \equiv 2 \overline{\mathbf{X}}+\overline{\mathbf{X}} \mathbf{M} \overline{\mathbf{X}}, \quad \overline{\mathbf{X}}_{\mu \rho} \equiv-\left(\mu^{2}-\rho^{2}\right) \overline{\mathbf{X}}-\mathbf{D D}^{\mathbf{T}}+$ $\mu \mathbf{A} \overline{\mathbf{X}}+\mu \overline{\mathbf{X}} \mathbf{A}^{\mathbf{T}}-\mathbf{A} \overline{\mathbf{R}} \mathbf{A}^{\mathbf{T}}$ and $\left[\bullet^{+}\right.$denotes the MoorePenrose inverse of matrix $[\bullet]($ see $[20])$.

\section{Proof:}

It is clear that equation (9) may be written as

$$
\begin{gathered}
{\left[\mathbf{B G P}+(\mathbf{A} \overline{\mathbf{R}}-\mu \overline{\mathbf{X}})\left(\mathbf{P} \mathbf{P}^{\mathbf{T}}\right)^{-1} \mathbf{P}\right][\mathbf{B G P}+(\mathbf{A} \overline{\mathbf{R}}} \\
\left.-\mu \overline{\mathbf{X}})\left(\mathbf{P} \mathbf{P}^{\mathbf{T}}\right)^{-1} \mathbf{P}\right]^{\mathbf{T}}=\mathbf{K K}^{\mathbf{T}},
\end{gathered}
$$

where $\mathbf{P P}^{\mathbf{T}} \equiv \overline{\mathbf{R}}$ and $\mathbf{K} \in \mathbf{R}^{\mathbf{n}_{\mathbf{x}} \times \mathbf{n}_{\mathbf{y}}}$ is defined by

$$
\overline{\mathbf{X}}_{\mu \rho}+(\mathbf{A} \overline{\mathbf{R}}-\mu \overline{\mathbf{X}}) \overline{\mathbf{R}}^{-1}\left(\overline{\mathbf{R}} \mathbf{A}^{\mathbf{T}}-\mu \overline{\mathbf{X}}\right)=\mathbf{K} \mathbf{K}^{\mathbf{T}}
$$

From (21), it can be found that the right-hand side of (22) is semi-positive definite. Hence, condition (19) will be immediately obtained. From Lemma 2.1 of [21], (21) is equivalent to

$$
\mathbf{B G}=\mathbf{K V P} \mathbf{P}^{-1}-(\mathbf{A} \overline{\mathbf{R}}-\mu \overline{\mathbf{X}})\left(\mathbf{P P}^{\mathbf{T}}\right)^{-1},
$$

where $\mathbf{V}$ is some orthogonal matrix. From the theory of generalized inverse [22], equation (23) must be solvable for $\mathbf{G}$, which is guaranteed if and only if

$$
\left(\mathbf{I}-\mathbf{B B}^{+}\right) \mathbf{K V}=\left(\mathbf{I}-\mathbf{B} \mathbf{B}^{+}\right)(\mathbf{A} \overline{\mathbf{R}}-\mu \overline{\mathbf{X}})\left(\mathbf{P} \mathbf{P}^{\mathbf{T}}\right)^{-1} \mathbf{P} .
$$

Since $\mathbf{P}$ is nonsingular, using Lemma 2.1 of [21] and (22), equation (24) is equivalent to

$$
\left(\mathbf{I}-\mathbf{B B}^{+}\right)\left[\mathbf{K K}^{\mathbf{T}}-(\mathbf{A} \overline{\mathbf{R}}-\mu \overline{\mathbf{X}}) \overline{\mathbf{R}}^{-1}\left(\overline{\mathbf{R}} \mathbf{A}^{\mathbf{T}}-\mu \overline{\mathbf{X}}\right)\right]\left(\mathbf{I}-\mathbf{B B}^{+}\right)=0 .
$$

From equation (22), we can find that (25) is equivalent to (20).

Using Theorem 1 and Definition 3 we know that if the necessary and sufficient conditions of Theorem 1 hold for a specified upper bound covariance matrix
$\overline{\mathbf{X}}$, then there exists some matrices $\mathbf{G}$ such that equation (9) is satisfied. When equation (9) is satisfied. performance constraints $<1>-<3\rangle$ can be achieved from Lemma 1. Here, it suggests that an assignment must be made for the diagonal entries of $\overline{\mathbf{X}}$ to satisfy the variance constraint $\langle 1\rangle$. From condition (20), the unknown off-diagonal entries of $\overline{\mathbf{X}}$ will characterize a nonlinear simultaneous equation. Using the well known numerical methods (such as Fixed-point iteration method and Newton's method [23]), we can obtain suitable off-diagonal entries of $\overline{\mathbf{X}}$ for satisfying (20) and $\overline{\mathbf{X}}=\overline{\mathbf{X}}^{\mathbf{T}}>0$. Finally, we must substitute the matrix $\overline{\mathbf{X}}$ into condition (19). If condition (19) is satisfied, then controller $\mathbf{G}$ will be obtained from the following theorem. Otherwise, the diagonal and offdiagonal entries of $\overline{\mathbf{X}}$ must be reassigned.

\section{Theorem 2}

If the conditions of Theorem 1 hold for a specified upper bound covariance matrix $\overline{\mathbf{X}}$, then the state feedback controller $\mathbf{G}$ which achieve this specified $\overline{\mathbf{X}}$ is given by

$$
\begin{aligned}
\mathbf{G} & =\mathbf{B}^{+}\left[\mathbf{K} \mathbf{V}_{\mathbf{k}}\left[\begin{array}{cc}
\mathrm{I} & 0 \\
0 & \mathbf{U}_{\mathrm{a}}
\end{array}\right] \mathbf{V}_{\mathbf{P}}^{\mathrm{T}} \mathbf{P}^{-1}-(\mathbf{A} \overline{\mathbf{R}}\right. \\
& \left.-\mu \overline{\mathbf{X}})\left(\mathbf{P} \mathbf{P}^{\mathrm{T}}\right)^{-1}\right]+\left(\mathbf{I}-\mathbf{B}^{+} \mathbf{B}\right) \tilde{\mathbf{Z}},
\end{aligned}
$$

where $\mathbf{K}$ is expressed as in equation (22), $\tilde{\mathbf{Z}} \in \mathbf{R}^{\mathbf{n}_{\mathbf{u}} \times \mathbf{n}_{\mathbf{y}}}$ is an arbitrary matrix, $\mathbf{U}_{\mathrm{a}} \in \mathbf{R}^{\left(\mathbf{n}_{\mathbf{y}}-\mathbf{r}_{\mathbf{k}}\right) \times\left(\mathbf{n}_{\mathbf{y}}-\mathbf{r}_{\mathbf{k}}\right)}$ is an arbitrary orthogonal matrix, $r_{k}=\operatorname{Rank}\left[\left(\boldsymbol{I}-\boldsymbol{B B}^{+}\right) \boldsymbol{K}\right]$, and $\mathbf{V}_{\mathbf{k}}$ and $\mathbf{V}_{\mathbf{p}}$ come from the SVD as follows:

$$
\begin{aligned}
& \left(\mathbf{I}-\mathbf{B B}^{+}\right) \mathbf{K}=\hat{\mathbf{U}} \boldsymbol{\Sigma} \mathbf{V}_{\mathbf{k}}^{\mathbf{T}}, \\
& \left(\mathbf{I}-\mathbf{B} \mathbf{B}^{+}\right)(\mathbf{A} \overline{\mathbf{R}}-\mu \overline{\mathbf{X}})\left(\mathbf{P} \mathbf{P}^{\mathbf{T}}\right)^{-1} \mathbf{P}=\hat{\mathbf{U}} \boldsymbol{\Sigma} \mathbf{V}_{\mathbf{p}}^{\mathbf{T}} .
\end{aligned}
$$

\section{Proof:}

From the proof of Theorem 1, it is known that the given $\overline{\mathbf{X}} D$ - $\gamma$-assignable if and only if there exists a solution $\mathbf{G}$ to (23) for some orthogonal matrix $\mathbf{V}$ satisfying (24). Using the theory of generalized inverses [22] and the Lemma 2.1 of [21], solution G of (23) has the forms described by (26). \#

From the above theorems we know that the first step in designing a state feedback controller to satisfy constraints $\langle 1\rangle-<3\rangle$ is to assign a suitable upper bound covariance matrix $\overline{\mathbf{X}}$ which satisfies the performance constraint $\langle 1\rangle$. Conditions (19) and (20) of Theorem 1 must also be achieved. If the necessary and sufficient conditions of Theorem 1 are satisfied, then the state feedback controller solution $\mathbf{G}$ will be obtained from (26) of Theorem 2. To achieve con- 
straint $\langle 4\rangle$, the necessary conditions for minimizing auxiliary entropy $\boldsymbol{I}_{u}(\boldsymbol{H}, \gamma)$ via state feedback controller (26) will be presented in the following theorem.

\section{Theorem 3}

If state feedback controller $\mathbf{G}$ defined by (26) minimizes the auxiliary entropy $\boldsymbol{I}_{\boldsymbol{u}}(\boldsymbol{H}, \gamma)$ with $\|\mathbf{H}(\mathbf{z})\|_{\infty}$ $\leq \gamma$, then there exists a scalar $\alpha \geq 0$ and matrices $\bar{X}=\bar{X}^{T}>0, N=N^{T} \geq 0$, such that (19)-(20) and the following conditions are simultaneously satisfied:

$$
\alpha\left(I-\gamma^{-2} \bar{X}\right)^{-1}+\rho^{2} N_{b}-Q_{1}^{T} N_{b} Q_{1}-Q_{2}^{T} N_{b} Q_{2}=0,
$$

where $\boldsymbol{N}_{b} \equiv\left(\boldsymbol{I}-\boldsymbol{B} \boldsymbol{B}^{+}\right) \boldsymbol{N}\left(\boldsymbol{I}-\boldsymbol{B} \boldsymbol{B}^{+}\right), \boldsymbol{Q}_{1} \equiv \boldsymbol{A}-\mu \boldsymbol{I}$ and $\boldsymbol{Q}_{2} \equiv \boldsymbol{A}+\boldsymbol{A} \overline{\boldsymbol{X}}\left(\gamma^{2} I-\overline{\boldsymbol{X}}\right)^{-1}$.

\section{Proof:}

By minimizing the auxiliary entropy $\boldsymbol{I}_{u}(\boldsymbol{H}, \gamma)$ through the state feedback controller $\mathbf{G}$ defined by (26), it is necessary for conditions (19)-(20) of Theorem 1 to be satisfied. To optimize $\boldsymbol{I}_{\boldsymbol{u}}(\boldsymbol{H}, \gamma)$ defined by (6), subject to the constraint (20), form the Lagrangian

$$
\begin{aligned}
\boldsymbol{J} & =-\alpha \gamma^{2} \ln \operatorname{det}\left(\boldsymbol{I}-\gamma^{-2} \overline{\boldsymbol{X}}\right)+\operatorname{tr} \boldsymbol{N}\left\{( \boldsymbol { I } - \boldsymbol { B } \boldsymbol { B } ^ { + } ) \left[-\left(\mu^{2}\right.\right.\right. \\
& \left.-\rho^{2}\right) \overline{\boldsymbol{X}}-\boldsymbol{D} \boldsymbol{D}^{T}+\mu \boldsymbol{A} \overline{\boldsymbol{X}}+\mu \overline{\boldsymbol{X}} \boldsymbol{A}^{T}-\boldsymbol{A}(2 \overline{\boldsymbol{X}} \\
& \left.\left.\left.+\overline{\boldsymbol{X}}\left(\gamma^{2} \boldsymbol{I}-\overline{\boldsymbol{X}}\right)^{-1} \overline{\boldsymbol{X}}\right) \boldsymbol{A}^{T}\right]\left(\boldsymbol{I}-\boldsymbol{B} \boldsymbol{B}^{+}\right)\right\},
\end{aligned}
$$

where the Lagrange multipliers $\alpha \geq 0$ and $N=N^{T} \geq 0$ are not both zero. By taking the partial derivatives [24] of (30) with respect to $N, \bar{X}$ and setting them to zero, it is easy to obtain the necessary conditions (20) and (29) for minimizing the auxiliary entropy $\boldsymbol{I}_{u}(\boldsymbol{H}$, $\gamma$.

\section{AN EXAMPLE}

Consider the linear discrete system (1) with the following parameters:

$$
\begin{aligned}
{\left[\begin{array}{c}
x_{1}(k+1) \\
x_{2}(k+1)
\end{array}\right] } & =\left[\begin{array}{cc}
0.15 & 0.08 \\
0 & -3.5
\end{array}\right]\left[\begin{array}{l}
x_{1}(k) \\
x_{2}(k)
\end{array}\right]+\left[\begin{array}{c}
0 \\
-2
\end{array}\right] u(k) \\
& +\left[\begin{array}{c}
0 \\
0.01
\end{array}\right] w(k) .
\end{aligned}
$$

It is assumed that the performance requirements have the following form

$$
\begin{aligned}
& <1>E\left[x_{1}(k)^{2}\right] \leq 0.5, \quad E\left[x_{2}(k)^{2}\right] \leq 0.1, \\
& <2>\|\mathrm{H}(\mathrm{z})\|_{\infty} \leq 0.8 \text { (i.e., } \gamma=0.8 \text { ), }
\end{aligned}
$$

$<3>\mu=0.5$ and $\rho=0.4$ for disk $\boldsymbol{D}(\mu, \rho)$.

Substituting $\mu=0.5, \rho=0.5$ and $\gamma=0.8$ into condition (20) and assigning $\tilde{x}_{22}=0.025$ and $\dot{x}_{12}=0$

$$
\text { for } \begin{aligned}
\mathbf{X} & =\left[\begin{array}{ll}
\overline{\boldsymbol{x}}_{11} & \overline{\boldsymbol{x}}_{12} \\
\overline{\boldsymbol{x}}_{12} & \overline{\boldsymbol{x}}_{22}
\end{array}\right] \text {, then the condition (20) becomes } \\
& -0.0375 \dot{x}_{11}^{2}+0.0099 \tilde{x}_{11}-2.0896 \times 10^{-4}=0
\end{aligned}
$$

Note that the left-hand side of the equation (35) is the $(1,1)$ element of matrix $\overline{\mathbf{X}}_{\mu \rho}$. Solving (35) we obtain $\hat{\boldsymbol{x}}_{11}=0.2416$, which satisfies constraint $<1>$. We may now obtain

$$
\bar{X}=\left[\begin{array}{cc}
0.2416 & 0 \\
0 & 0.025
\end{array}\right] \text {. }
$$

It is easy to check that performance constraint $<1>$ and condition (20) are satisfied. Furthermore, by substituting the above matrices into (18), it is easy to find that condition (19) is also satisfied.

Since conditions (19)-(20) of Theorem 1 are satisfied, from (22) and $\mathbf{P P}^{\mathbf{T}}=\overline{\mathbf{R}}$ we can obtain matrices $\mathbf{P}$ and $\mathbf{K}$ as follows

$$
P=\left[\begin{array}{cc}
0.7937 & 0 \\
0 & 0.2259
\end{array}\right], K=\left[\begin{array}{cc}
0.0378 & 0 \\
0 & 0.0267
\end{array}\right] \text {. }
$$

Furthermore, the matrices $\mathbf{V}_{\mathbf{k}}$ and $\mathbf{V}_{\mathbf{p}}$ can result from equation (27) and (28) by using SVD,

$$
V_{k}=\left[\begin{array}{ll}
1 & 0 \\
0 & 1
\end{array}\right], V_{p}=\left[\begin{array}{cc}
0.8783 & -0.4782 \\
-0.4782 & 0.8783
\end{array}\right] \text {. }
$$

From (26), the state feedback control gain matrix $\mathbf{G}$ can be obtained.

$$
G=\left[\begin{array}{ll}
0.008 & -1.8206
\end{array}\right] .
$$

From the simulated responses (Fig. 1 and Fig. 2 ), we may obtain the variances of individual system states: $E\left[x_{1}(k)^{2}\right]=6.9183 \times 10^{-7}$ and $E\left[x_{2}(k)^{2}\right]=1.0067$ $\times 10^{-4}$. It can be found that the performance constraint (32) is satisfied. Substituting the state feedback controller (37) into the discrete $\mathbf{H}_{\infty}$ norm definition (Definition 1), we can obtain $\|H(z)\|_{\infty}=0.0011$ from $\boldsymbol{w}(\boldsymbol{k})$ to $x_{1}(\boldsymbol{k})$ and $\|H(z)\|_{\infty}=0.0116$ from $\boldsymbol{w}(\boldsymbol{k})$ to $\boldsymbol{x}_{2}(\boldsymbol{k})$. It is obvious that the $\mathbf{H}_{\infty}$ norm of the closed-loop transfer function satisfies the performance requirement (33). Calculating the eigenvalues of the closedloop matrix $\hat{\mathbf{A}}$, we can find that the closed-loop system poles are $0.1456 \pm 0.0356 i$, which are located in the specified disk $D(\mu, \rho)$. From the above descriptions, it is shown that the state feedback controller (37) drives the linear discrete system (31) to achieving the multiple performance requirements $\langle 1\rangle-\langle 3\rangle$. 


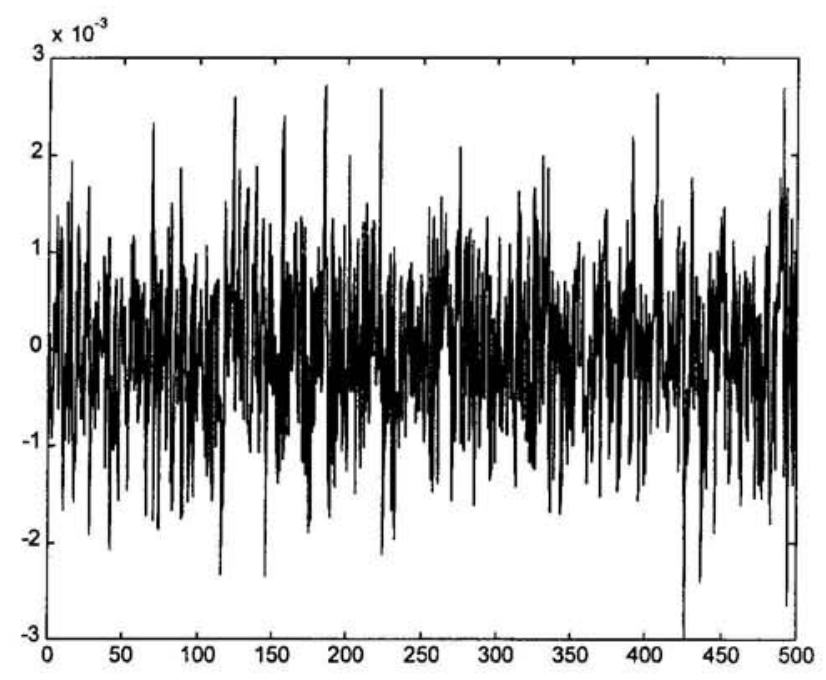

Fig. 1. Steady state response of state $x_{1}(k)$.

From Theorem 3, we know that if the state feedback controller (37) minimizes the auxiliary entropy $\boldsymbol{I}_{\boldsymbol{u}}(\boldsymbol{H}$, $\gamma$ ) (i.e., the constraint $\langle 4\rangle$ is satisfied), then there exists a scalar $\alpha \geq 0$ and matrices $\bar{X}=\bar{X}^{T}>0, N=N^{T}$ $\geq 0$ such that conditions (19), (20) and (29) are all satisfied. To achieve constraint $\langle 4\rangle$, we choose $\alpha=$ 0 and substitute $\overline{\boldsymbol{X}}$ defined in (36) into condition (29). We may then conclude that the $(1,1)$ element of matrix $N$ must be zero and the other elements must be commensurately chosen for $\boldsymbol{N}=\boldsymbol{N}^{T} \geq 0$. Placing $\overline{\boldsymbol{X}}$ defined in (36) into (6), we obtain the minimum upper bound entropy $I_{u}(H, \gamma)=0.3289$.

\section{CONCLUSIONS}

This paper has provided a solution of multiple constrained state feedback controller design problems for linear discrete systems. The performance constraints considered in this paper include regional pole placement, $\mathbf{H}_{\infty}$ norm constraints, individual variance constraints and minimum auxiliary entropy. We first introduced a Riccati-like equation whose solution gives an upper bound of the state covariance matrix. Based on this Riccati-like equation, the conditions and solutions of the multiple constrained state feedback controller design problems are derived through the SVD theory, the generalized inverse theory, and the Lagrange multiplier technique.

\section{ACKNOWLEDGEMENTS}

The author would like to express his sincere gratitude to two anonymous reviewers who gave me some constructive comments, cirticisms and suggestions. This work was supported by the National

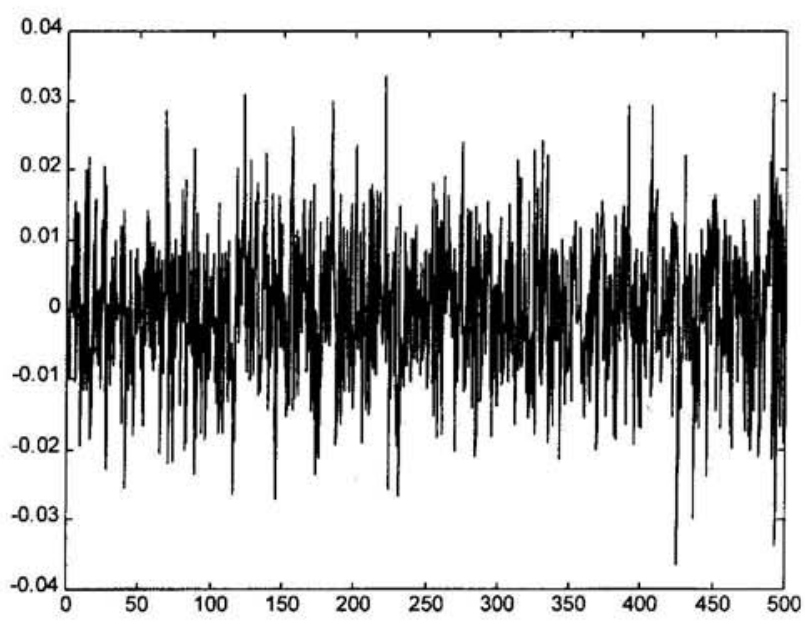

Fig. 2. Steady state response of state $x_{2}(k)$.

Science Council of the Republic of China, under contract NSC87-2213-E-019-012.

\section{REFERENCES}

1. Mustafa, D. and Glover, K., Minimum Entropy $\mathbf{H}_{\infty}$ Control, Lecture Notes in Control and Information Sciences 146, Springer-Verlag, Heidelberg (1990).

2. Iglesias, P.A. and Mustafa, D., "State-Space Solution of the Discrete-Time Minimum Entropy Control Problem Via Separation," IEEE Trans. Auto. Control, Vol. 38 , No. 10, pp. 1525-1530 (1993).

3. Iglesias, P.A., "An Entropy Formula for Time-Varying Discrete-Time Control Systems," SIAM J. Control and Optimization, Vol. 34, No. 5, pp. 1691-1706 (1996).

4. Mustafa, D. and Bernstein, D.S., "LQG Cost Bounds in Discrete-Time $\mathbf{H}_{2} / \mathbf{H}_{\infty}$ Control," Trans. of the Institute of Measurement and Control, Vol. 13, No. 5, pp. 269-275 (1991).

5. Whittle, P., Risk-Sensitive Optimal Control, John Wiley and Sons, New York (1990).

6. Geromel, J.C., Peres, P.L.D. and de Souza, S.R., " $\mathbf{H}_{2}$ Guaranteed Cost Control for Uncertain Discrete-Time Linear Systems," Int. J. Control, Vol. 57, No. 4, pp. 853-864 (1993).

7. Kaminer, I., Khargonekar, P.P. and Rotea, M.A., "Mixed $\mathbf{H}_{2} / \mathbf{H}_{\infty}$ Control for Discrete-Time Systems via Convex Optimization," Automatica, Vol. 29, No. 1, pp. 57-70 (1993).

8. Haddad, W.M., Bernstein, D.S. and Mustafa, D., "Mixed-Norm $\mathbf{H}_{2} / \mathbf{H}_{\infty}$ Regulation and Estimation : The Discrete-Time Case," System \& Control Letters, Vol. 16, pp. 235-247, 1991.

9. Gahinet, P., Nemirovski, A. and Laub, A., LMI Control Toolbox: for use with Matlab, Mathworks, Inc. (1995). 
10. Haddad, W.M. and Bernstein, D.S., "Controller Design with Regional Pole Constraints," IEEE Trans. Auto. Control, Vol. 37, No. 1, pp. 54-69 (1992).

11. Garcia, G. and Bernussou, J., "Pole Assignment for Uncertain Systems in a Specified Disk by State Feedback," IEEE Trans. Auto. Control, Vol. 40, No. 1, pp. 184-190 (1995).

12. Moheimani, O.R. and Petersen, I.R., "Quadratic Guaranteed Cost Control with Robust Pole Placement in a Disk," IEE Proceeding, Part D, Control Theory and Applications, Vol. 143, No. 1, pp. 37-43 (1996).

13. Tsay, S.C., Fong, I.K. and Kuo, T.S., "D-stability Analysis for Discrete Optimal Regulator," ControlTheory and Advanced Technology, Vol. 6, No. 2, pp. 237-246 (1990).

14. Lee, C.H., Li, T.H.S. and Kung, F.C., "D-stability Analysis for Discrete System with a Time Delay," System \& Control Letters, Vol. 19, pp. 213-219 (1992).

15. Skelton, R.E., Dynamic Systems Control: Linear Systems Analysis and Synthesis, Wiley, New York (1988).

16. Chung, H.Y. and Chang, W.J., "Covariance Control with Variance Constraints for Continuous Perturbed Stochastic Systems," Systems \& Control Letters, Vol. 19, No. 5, pp. 413-417 (1992).

17. Chang, W.J. and Chung, H.Y., "Upper Bound Covariance Control of Discrete Perturbed Systems," Systems \& Control Letters, Vol. 19, No. 6, pp. 493-498 (1992).

18. Chang, W.J. and Chung, H.Y., "A Study of $\mathbf{H}_{\infty}$ Norm and Variance Constrained Design Using Dynamic Output Feedback for Linear Discrete Systems," Int, J. Control, Vol. 57, No. 2, pp. 473-483 (1993).

19. Chang, W.J. and Chung, H.Y., "H $\mathbf{H}_{\infty}$ Norm and Variance Constrained Design via Dynamic Controllers for Linear Discrete Systems with Structured Real Parameter Uncertainty," J. of Chinese Institute of Electrical Engineering, Vol. 3, No. 2, pp. 139-146 (1996).

20. Horn, R.A. and Johnson, C.R., Matrix Analysis, Cam- bridge University Press (1985).

21. Xu, J.H. and Skelton, R.E., "An Improved Covariance Assignment Theory for Discrete Systems," IEEE Trans. Auto. Control, Vol. 37, No. 10, pp. 1588-1591 (1992).

22. Rao, C.R. and Mitra, S.K., Generalized Inverse of Matrices and Its Applications, Wiley, New York (1971).

23. Mathews, J.H., Numerical Methods for Computer Science, Engineering, and Mathematics, Prentice-Hall Int., Inc. (1987).

24. Geering, H.P., "On Calculating Gradient Matrices," IEEE Trans. Auto. Control, Vol. 21, No. 8, pp. 615 616 (1976).

線性間断系統滿足多重行為限制及 最小化摘之強健狀態回授控制器設 計

張 文 哲

國立台管海洋大學輪機工程技街系副教授

摘 要

本篇就文將䦐明一個能夠满足最小化輔助熇 的狀態回授控制器存在性之設計問題。此一狀態回 授控制器將使線性間断系統满足多重行為限制, 這 些系統行為需求包括個别狀態方差限制、 $\mathbf{H}_{\infty}$ 模限 制及區域極點指定限制。利用奇異值分解技術及廣 義逆求解理論, 我們便可求得此一狀態回授控制器 之解, 以使前述之三項行為限制達到要求。本篇論 文理論的有效性我們將用一個数值例子來加以說 明。 